\title{
CARCINOMA IN SITU DEL CUELLO UTERINO
}

\section{CARCINOMA INTRAEPITELIAL - 233 Casos - Control y Seguimiento}

ESTUDIO DE 233 CASOS EN EL HOSPITAL UNIVERSITARIO SAN VICENTE DE PAUL DE MEDELLIN, ENTRE ENERO DE 1958 Y DICIEMBRE DE 1971.

Dr. Eduardo Baquero B.*

Dr. Julio C. Tuberquia C.**

Dra. Constanza Díaz de Calle***

\section{Introducción}

El diagnóstico de cáncer in situ se hace cada vez más frecuente entre nosotros debido principalmente al auge de la citología como medio de detección del cáncer genital. La disponibilidad de este material ha llevado a refinamientos diagnósticos y a encontrar casos más incipientes de esta entidad que antes era un diagnóstico de excepción.

Ya en 1956 en un importante trabajo sobre este tema (1) se llama la atención sobre el gran volumen de publicaciones al respecto y a la importancia de la toma de la citología en todas las pacientes, cualquiera que sea su enfermedad, edad, embarazo, etc. con el fin de detectar el carcinoma pre-invasor. Otro autor (2) dá cierta prioridad a determinadas pacientes como son: casadas, multíparas, o a la de 30 o más años.

En nuestro medio es claro este aumento de carcinoma in situ y cada vez es mayor el porcentaje de casos encontrados; pensamos que en un futuro no muy lejano disminuyan en una forma proporcional los casos de cáncer invasor del cérvix en favor del pre-invasor.

Papel importante en la detección del cáncer genital en general han sido las campañas de planificación familiar que han tratado de cubrir a las personas de bajos recursos que acuden a los centros de Salud y a los programas de post-parto de las diferentes clínicas de la ciudad, como el Hospital Universitario San Vicente de Paúl y la Clínica de Maternidad de Medellín Luz Castro de Gutiérrez, que atienden clientela de escasos recursos económicos, donde parece que es más frecuente el carcinoma de cérvix (3). De rutina se les toma a estas pacientes una citología oncológica,

* Residente de tercer año, Departamento de Obstetricia y Ginecología, Facultad de Medicina, Universidad de Antioquia.

** Profesor Auxiliar, Departamento de Obstetricia y Ginecología, Facultad de Medicina, Universidad de Antioquia.

*** Profesor Auxiliar, Departamento de Patología, Facultad de Medicina, Universidad de Antioquia. 
cuando asisten a un centro de planificación familiar.

Aunque todavía no hay unanimidad de criterios respecto a algunas diferencias histopatológicas que separan la displasia epitelial severa del carcinoma in situ (4), sí hay una inmensa mayoría de patólogos con experiencia que pueden trabajar en este campo con precisión. La conducta terapéutica en las dos entidades mencionadas es prácticamente la misma.

Se van a encontrar algunas conductas de tratamiento muy diversas dado el tiempo que abarca nuestro trabajo; es evidente que los procedimientos terapéuticos han mejorado y ya se tiene una visión más clara de la entidad.

Se consideran también en este trabajo algunas pacientes con carcinoma in situ y embarazo sobre las cuales se dsicutirán los diversos procedimientos que se llevaron a efecto y las discrepancias que hay en la literatura respecto al tratamiento más adecuado.

Hay que hacer notar a la paciente que esta lesión intraepitelial, bien tratada tiene una curación del $100 \%$, para no crear en ella temores infundados al decirle que tiene un cáncer in situ, porque esta palabra puede llevar a confusión y alarma, que no tiene razón de ser, ni mucho menos, cuando son pacientes con siquismo lábil y fácilmente influenciable por palabras mal dichas y por lo tanto también mal interpretadas.

Hay un acuerdo especial en cuanto a que el carcinoma in situ representa una forma pre-invasiva del carcinoma del cérvix, pero el verdadero significado de la displasia ha sido una fuente de controversia (4).
Algunos autores prefieren no utilizar la terminología diferencial entre carcinoma in situ y displasia; usan mejor el término "neoplasia cervical intraepitelial" graduándola de 1 a 4, donde el 1 correspondería a displasia leve y el 4 al carcinoma in situ (4).

Es interesante también anotar que repetidamente en las biopsias de carcinoma invasor de cérvix se encuentran las tres fases sucesivas de los cambios morfológicos que hemos venido hablando: displasia, carcinoma intraepitelial y carcinoma invasor (3).

\section{Historia}

En 1886 Sir John Williams describió la forma no invasora del carcinoma del cuello uterino (5). Entre nosotros fue el Doctor Jordan quien publicó uno de los primeros trabajos al respecto (6) y trae la siguiente definición de carcinoma in situ: "Es la lesión histopatológica irreversible del cuello uterino en la cual el espesor de la capa epitelial, es una o varias zonas, es reemplazada por células microscópicamente malignas, pero que no muestran penetración invasora de la membrana basal del estroma subyacente"

Algunos autores especialmente los alemanes no creen que el carcinoma intraepitelial sea tan maligno (7) debido a la observación de algunos casos que han desaparecido sin tratamiento, y algunos otros que no han pasado del estado in situ por muchos años de seguimiento; estos autores llaman a este carcinoma in situ "epitelio atípico o epitelio inquieto". Pero estudios de otros autores han comprobado que estos carcinomas in situ sí pueden avanzar a carcinoma invasor y aconsejan tratar a todas las pacientes a quienes se les encuentre esta neoplasia pre-invasora $(3,7,8)$. 
Los trabajos de Petersen y FunckBrentano (9) demostraron que el seguir pacientes con carcinoma in situ sin efectuarles ningún tratamiento, aproximadamente la tercera parte desarrollaban carcinoma invasor en 9 a 10 años.

En el mismo orden de ideas ha evolucionado la conducta terapéutica, la cual es cada vez más radical $(1,10$, $11,12,13,14,15,16,17,18,19)$, en el sentido de hacer histerectomía total y resección de un manguito vaginal de por lo menos $2 \mathrm{cms}$. y no dejando a la paciente con una conización solamente como tratamiento; debemosa clarar que hay excepciones a la regla anterior como son aquellas pacientes jóvenes que desean tener más hijos $u$ otras pacientes que por su idiosincracia personal no quieren que su matriz les sea extraída debido a que les crearía conflictos de orden familiar tales como el posible abandono del hogar por el marido al saber que su esposa no tiene útero (20), o pacientes muy obesas o cardiópatas, etc. donde no está indicada ninguna intervención quirúrgica mayor.

\section{Objetivos}

El propósito de este trabajo es:

1. Analizar los métodos de detección, diagnóstico, tratamiento y el seguimiento de pacientes con carcinoma in situ del cérvix en el Hospital Universitario San Vicente de Paúl de Medellín durante los 13 últimos años.

2. Comparar los porcentajes de carcinoma in situ del cérvix uterino con el carcinoma invasor del mismo órgano, en el Hospital Universitario San Vicente de Paúl de Medellín.

3. Discutir el papel que desempeñe la conización para excluir carcinoma invasor en los casos en que se ha diagnosticado carcinoma in situ por biopsia múltiple.

4. Hacer notar la alta incidencia de carcinoma residual en el cuello postcono. Por lo tanto recalcar que el tratamiento definitivo es la Histerectomía total y resección del manguito vaginal.

5. Hacer notar la importancia que tiene diagnosticar el carcinoma de cérvix en sus estados más incipientes, por lo tanto la gran importancia de citología exfoliativa como método exploratorio.

\section{Material y Método}

La base de nuestro trabajo la constituyen 233 pacientes tratadas en dos diferentes períodos: un primer grupo retrospectivo que abarca de Enero de 1958 a Enero de 1968; y otro segundo grupo prospectivo que va desde Febrero de 1968 a Diciembre de 1971, para dar un total de 13 años de estudio. Todas las pacientes son tomadas de la consulta externa, sección de Ginecología, del Hospital Universitario San Vicente de Paúl y fureon manejadas por los miembros del Staff de profesores y residentes; este servicio ginecológico especializado es el único que funciona en la ciudad para pacientes de caridad al cual son remitidas gran número de ellas de los centros de salud y de otras ciudades.

Los estudios de Anatomía Patológica fueron revisados por el mismo patólogo para unificar criterios diagnósticos.

Las pacientes se estudiaron teniendo la mayoría la siguiente secuencia: A todas ellas se les hizo citología cérvico-vaginal según la técnica de $\mathrm{Pa}$ panicolaou que está clasificada en grados de I a $V$; consideramos citología positiva los grados IV y $\mathrm{V}$ a los 
GRAFICO N2I

DISTRIBUCION POR CADA DOS AÑOS DE DIAGNOSTICOS DE CA. IN-SITU. HOSPITAL UNIVERSITARIO SAN VICENTE DE PAUL. MEDELLIN 1.958-1.97I.

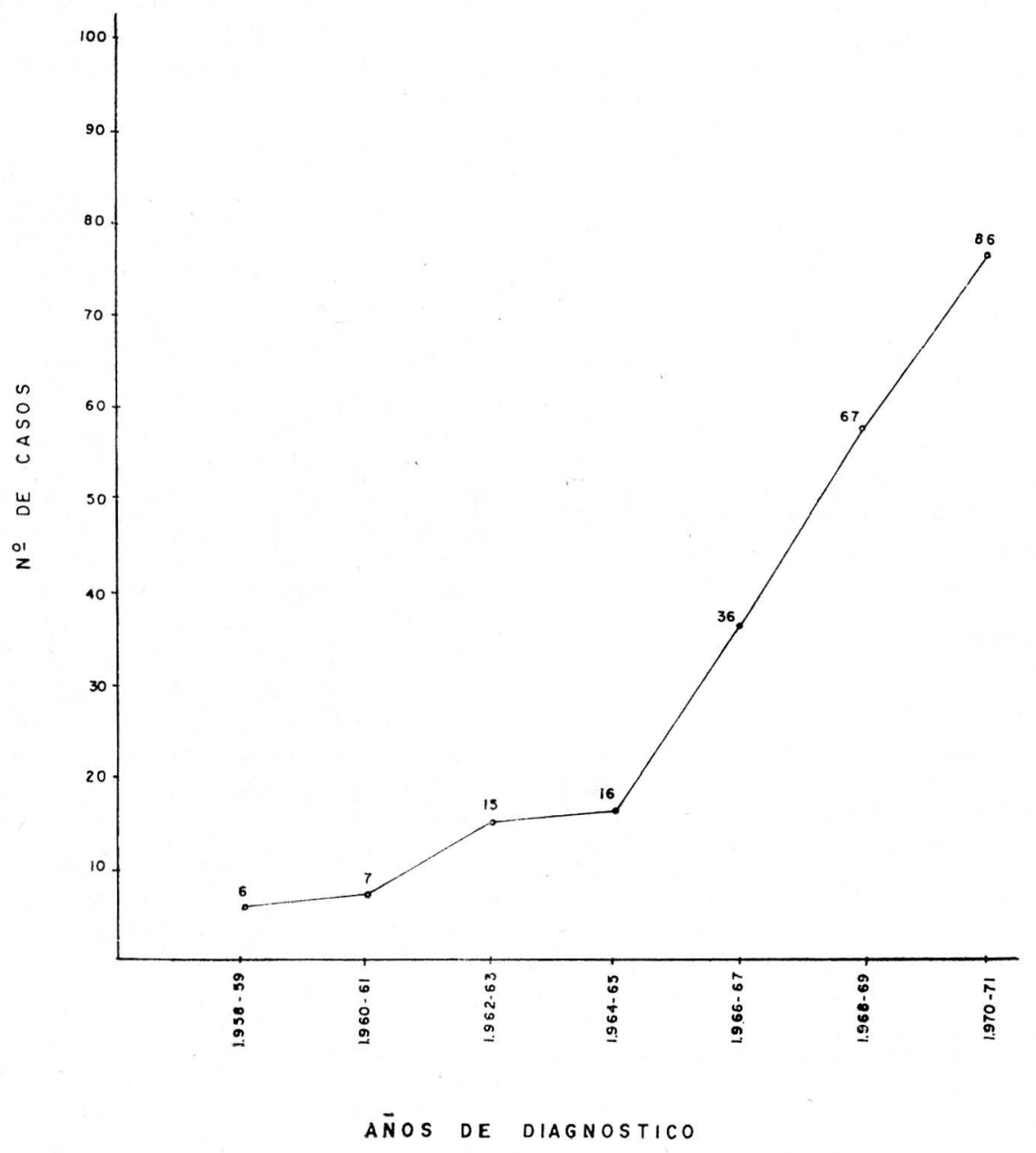


DISTRIBUCION POR EDAD Y AÑOS DE DIAGNOSTICOS DE 233 PACIENTES CON CA. CERVICAL IN SITU. HOSPITAL UNIVERSITARIO SAN VICENTE DE PAUL. MEDELLIN $1.958-1971$.

\begin{tabular}{|c|c|c|c|c|c|c|c|c|c|}
\hline \multirow{2}{*}{ EDAD } & \multicolumn{3}{|c|}{$A \bar{N}$ OS } & DI & GNOS & co & & \multirow{2}{*}{ TOTAL } & \multirow{2}{*}{$\%$} \\
\hline & $\begin{array}{l}1.958 \\
1.959\end{array}$ & $\begin{array}{l}1.960 \\
1.961\end{array}$ & $\begin{array}{l}1.962 \\
1.963\end{array}$ & $\begin{array}{l}1.964 \\
1.965\end{array}$ & $\begin{array}{l}1.966 \\
1.967\end{array}$ & $\begin{array}{l}1.968 \\
1.969\end{array}$ & $\begin{array}{l}1.970 \\
1.971\end{array}$ & & \\
\hline $20-29$ & 1 & 0 & 0 & I & 2 & 6 & 2 & 12 & - \\
\hline $30-39$ & 1 & 4 & 4 & 4 & 10 & 23 & 41 & 87 & - \\
\hline $40-49$ & 4 & 1 & 7 & 6 & 17 & 31 & 37 & 103 & - \\
\hline $50-59$ & 0 & 2 & 2 & 3 & 5 & 7 & 4 & 23 & - \\
\hline 60 y más & 0 & 0 & 2 & 2 & 2 & 0 & 2 & 8 & - \\
\hline TOTAL & 6 & 7 & 15 & 16 & 36. & 67 & 86 & 233 & 10000 \\
\hline
\end{tabular}

cuales se les tomó biopsia en cuatro cuadrantes o se hizo biopsia múltiple (6 ó más fragmentos), en algunas con previa prueba de Schiller. En las citologías grados III tratadas que vuelven a dar el mismo grado III en tres ocasiones en total, se efectúa biopsia con la misma técnica anterior.

Aunque varios autores informan que la colposcopia tiene gran importancia en la localización de las zonas del cérvix para toma rla biopsia selectiva $(21,22,23,24)$ nosotros no usamos colposcopio en nuestro traba- jo sino que nos valimos de la prueba de Schiller en los casos que creímos necesarios, la que nos dá una prceisión que puede compararse a la colposcopia según nuestra opinión y la de otros trabajos (5).

Aumento del diagnóstico del carcinoma in situ. For las mismas consideraciones anteriores referentes a la importancia de la citología y al incremento de la toma de ella ha aumentado el diagnóstico del carcinoma pre-invasor del cérvix (Gráfica № 1 ). En esta gráfica vemos como de 6 casos encontrados en los años de 1958 
EDAD DE 233 PACIENTES CON CA. IN-SITU. HOSPITAL UNIVERSITARIO SAN VICENTE DE PAUL. MEDELLIN $1.958-1.971$

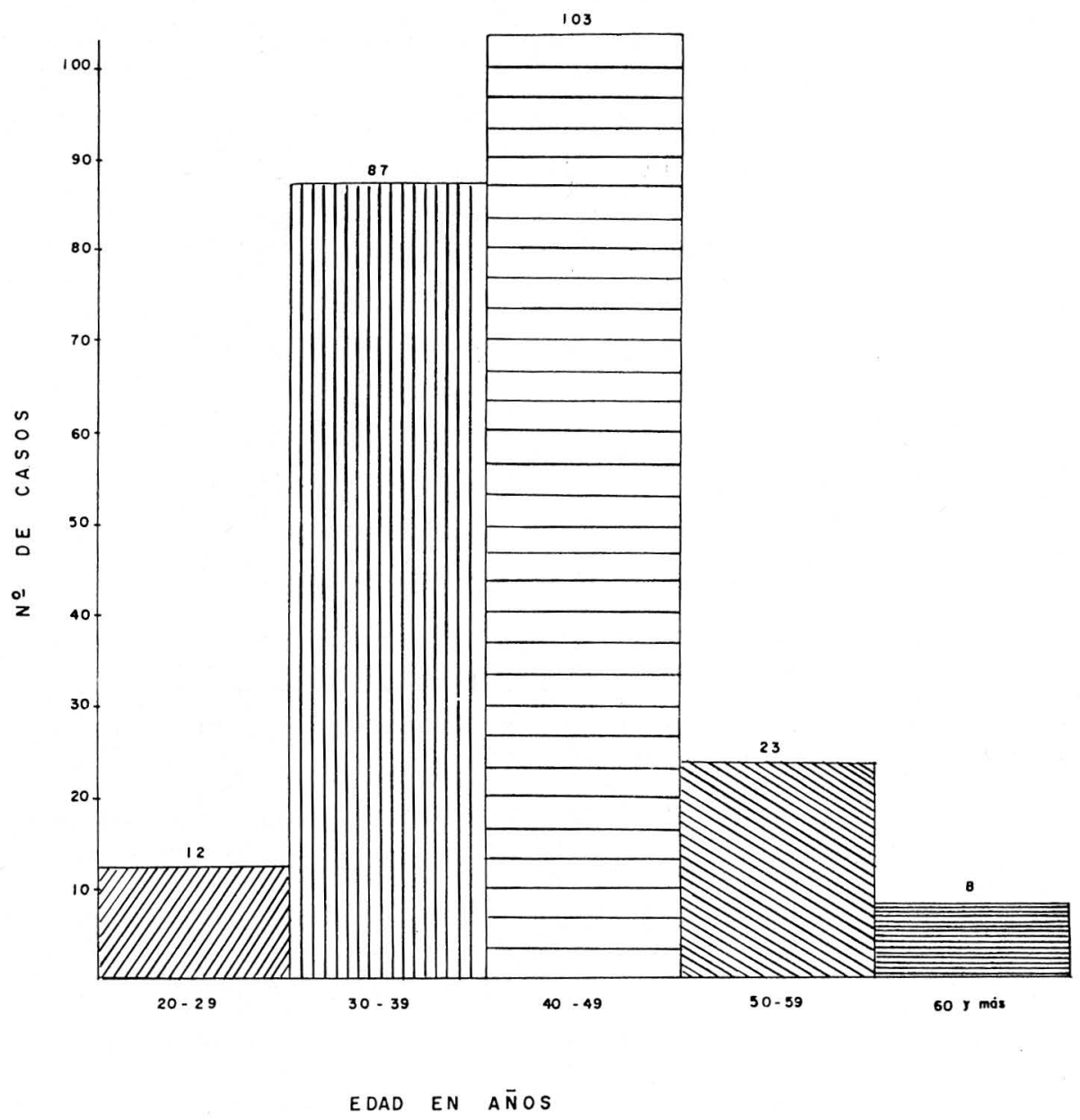


y 1959, pasamos a 86 casos diagnosticados en los últimos años del estudio (1970 y 1971). El Cuadro $N$ :1 y la Gráfica № 2 muestran que la edad de mayor frecuencia es la quinta década, destacándose de todas maneras como edades frecuentes las comprendidas entre 30 y 49 años. Si analizamos el hecho de que la displasia es una lesión previa al carcinoma in situ que se presenta siempre con más frecuencia en la etapa sexual activa, daría la posibilidad de que los estrógenos desempeñaran un papel importante en su producción $(3,25)$. Creemos nosotros que no es que esté au- mentando la frecuencia del carcinoma in situ, sino que se está buscando más y las pacientes puerperales tienen más acceso a los servicios médicos de reciente instalación y se ha creado en los médicos la conciencia de la toma de la citología de rutina en los centros generales de salud.

Carcinoma invasor del cérvix y carcinoma in situ. En nuestra consulta ginecológica hemos notado también un incremento de pacientes con carcinoma invasor del cérvix; éste posiblemente se deba a una mejor organización y al hecho de que hace 7

COMPARACION EN DIVERSOS AÑOS DEL CA. INTRAEPITELIAL CON RELACION AL CA. INVASOR POR ESTADOS CLINICOS. HOSPITAL UNIVERSITARIO SAN VICENTE DE PAUL. MEDELLIN $1.958-1.971$

\begin{tabular}{|c|c|c|c|c|c|c|c|c|c|c|}
\hline \multirow{2}{*}{$\begin{array}{c}\text { CARCINOMA } \\
\text { ESTADOS } \\
\text { CLINICOS }\end{array}$} & \multicolumn{9}{|c|}{ A N OS } \\
\cline { 2 - 11 } & 1.958 & 1.962 & 1.964 & 1.965 & 1.966 & 1.967 & 1.968 & 1.969 & 1.970 & 1.971 \\
\hline I & 3 & 7 & 9 & 7 & 11 & 24 & 29 & 39 & 38 & 48 \\
II & 23 & 28 & 10 & 23 & 14 & 19 & 35 & 43 & 33 & 37 \\
III & 58 & 47 & 70 & 80 & 54 & 79 & 65 & 84 & 77 & 73 \\
\hline IV & 16 & 12 & 9 & 9 & 4 & 9 & 5 & 2 & 1 & 2 \\
\hline
\end{tabular}


años funciona una consulta específica de oncología. Si vamos a relacionar estos dos tipos de tumores por años de aparición de diagnóstico, vemos que las curvas van en ascenso a medida que se hace más reciente la recopilación de los datos (Gráfica Ni 3 y Cuadro № 2). Algunos autores señalan que el tiempo para que un carcinoma in situ pase a carcinoma invasor es de aproximadamente 10 años $(9,3)$, y para que una displasia se convierta en carcinoma in situ la demora son 7 años $(4,26)$.

Por lo tanto no se justifica, al encontrar una displasia, dejarla evolucionar sin tratamiento.

Encontramos en el Cuadro № 2 y en la Gráfica № 4 varios datos que indican una disminución de los grados más avanzados de carcinoma en favor de los meses avanzados en los últimos años; éste posiblemente nos está indicando que ya los casos nos so nremitidos más precozmente, que la gente está motivándose rápidamente a consultar y también al incremento que ha tenido la consulta externa en los últimos años, pero todavía el espacio que separa el carcino$\mathrm{ma}$ in situ del carcinoma invasor (Gráfica № 3) es bastante amplio y debemos reducirlo, especialmente a expensas de la disminución de los casos de carcinoma invasor. No hicimos una división por edades de aparición del carcinoma invasor porque este no era nuestro objetivo; pero todos los autores están de acuerdo que la edad de aparición del carcinoma invasor es mayor que la del carcinoma in situ (27), siguiendo también al orden cronológico y la sucesión de los acontecimientos morfológicos en el cérvix hasta llegar al estado más avanzado $(3,4)$.

No están analizados en el Cuadro № 2 los años de 1959, 1960, 1961 y
1963 en lo que se refiere a carcinoma invasor debido a que los datos estadísticos en esos años no están muy completos.

\section{Base del diagnóstico del Carcino-} ma In Situ. En el Cuadro № 3 encontramos que a la mayoría de las pacientes se les hizo biopsia y como para precisar el diagnóstico $(81,12 \%)$ y al resto $(18,88 \%)$ sólo se les hizo biopsia múltiple.

En algunas de las pacientes se pasó directamente de la biopsia a la histerectomía sin la etapa intermedia de la conización. Algunos autores $(28,29)$ están de acuerdo en que el cono se puede obviar para disminuir la morbilidad y los costos extras que ésta intervención conlleva.

Motivo de Consulta. Llama la atención en el Cuadro № 4 que la sintomatología más frecuente fue el flujo y la hemorragia vaginal $(65,14 \%)$; pero también es importante el gran número de pacientes asintomáticas que presentaron carcinoma in situ $(26,60 \%)(30)$; este gran número de pacientes asintomáticas que suman algo más de la cuarta parte del total se debe a los casos encontrados en las consultas de planificación familiar donde la paciente acude, no por problemas ginecológicos especiales, sino por otra causa. Otro pequeño grupo de estas pacientes lo constituyen aquellas que rutinariamente se hacen tomar citología anual para estar seguras de que no tienen cáncer $(2,3)$. En el mismo cuadro se encuentran que los casos de prolapso gential con carcinoma in situ son escasos, lo cual está de acuerdo con la idea aceptada generalmente.

Aspecto clínico del cuello uterino. Un alto porcentaje de los cuellos se encontraban erosionados (62,23\%); llaman la atención que la tecerra par- 
GRAFICO N2 3

COMPARACION ENTRE CA. IN-SITU Y CA. INVASOR DEL CERVIX, POR AÑOS DEL DIAGNOSTICO. HOSPITAL UNIVERSITARIO SAN VICENTE DE PAUL MEDELLIN L958-1.97I

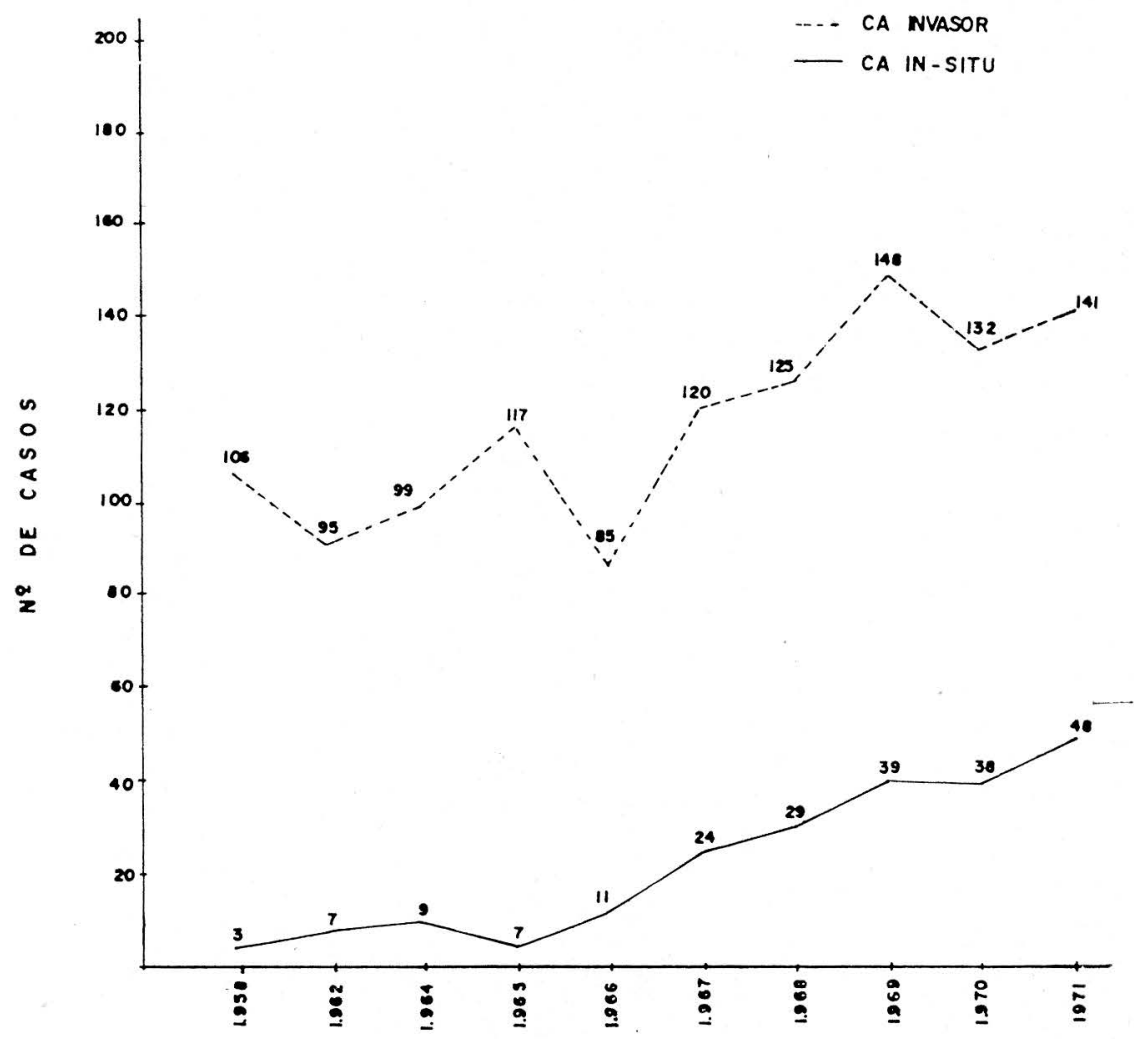

AÑOS DEL DIAGNOSTICO 


\section{GRAFICO N24}

COMPARACION GRAFICA EN DIFERENTES AÑOS DEL CARCINOMA INTRAEPITELIAL E INVASOR POR ESTADOS CLINICOS. HOSPITAL UNIVERSITARIO SAN VICENTE DE PAUL. MEDELLIN 1958-1.97I

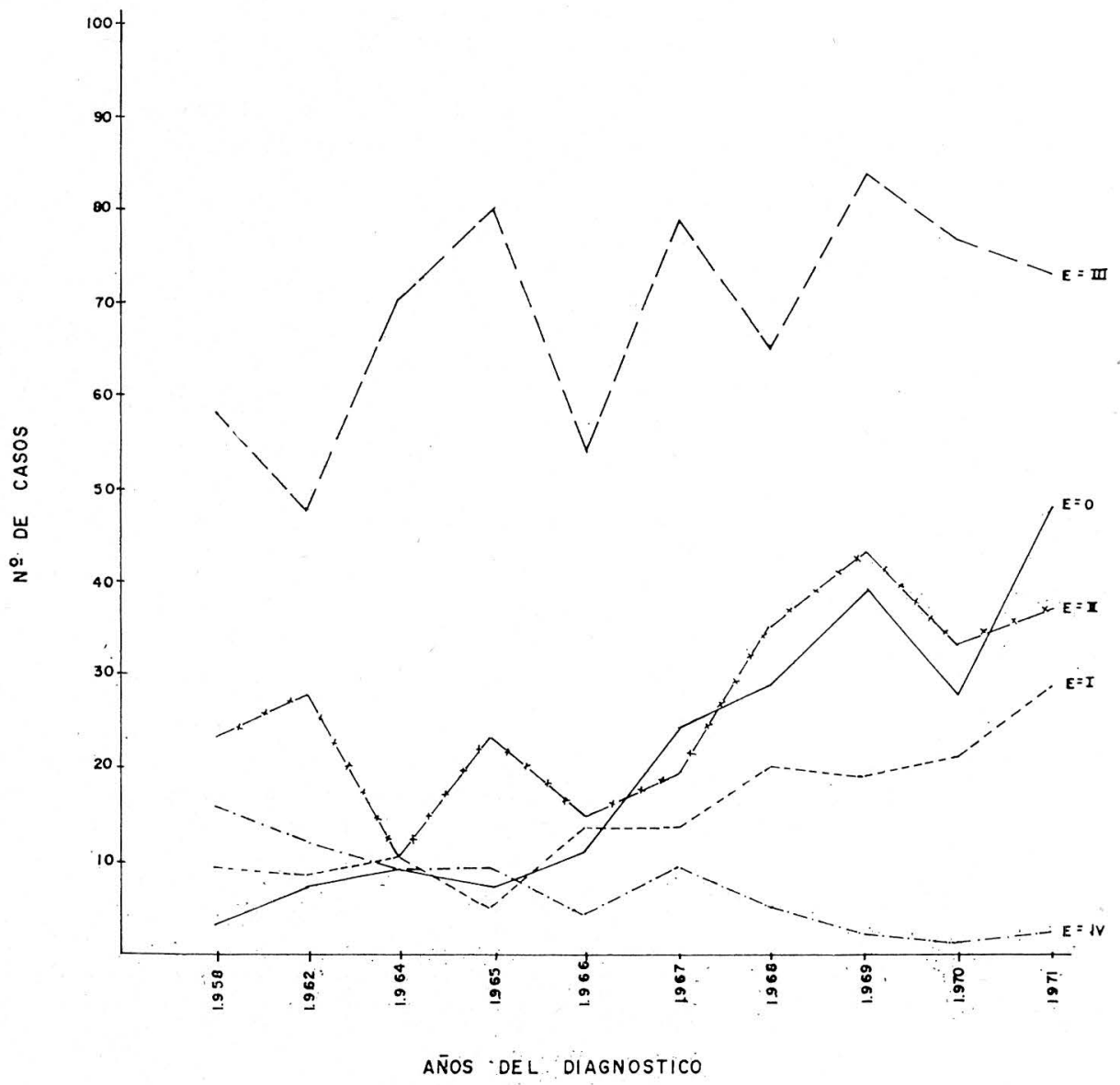




\section{CUADRO N2 3}

BASE DEL DIAGNOSTICO DE 233 CASOS DE CARCINOMA CERVICAL IN-SITU-HOSPITAL UNIVERSITARIO SAN VICENTE DE PAUL MEDELLIN 1.958-1.971.

\begin{tabular}{|c|c|c|c|c|c|}
\hline \multirow{2}{*}{$A \bar{N} O S$} & \multicolumn{5}{|c|}{ DIAGNOSTICO } \\
\hline & BIOPSIA & SOLAMENTE & BIOPSIA & Y C OND. & $T O T A L$ \\
\hline 1. 958 & & 2 & & 1 & 3 \\
\hline 1.959 & & 1 & & 2 & 3 \\
\hline 1.960 & & 1 & & 4 & 5 \\
\hline 1.961 & & 0 & & 2 & 2 \\
\hline 1.962 & & 3 & & 4 & 7 \\
\hline 1. 963 & & 0 & & 8 & 8 \\
\hline 1. 964 & & 3 & , & 6 & 9 \\
\hline 1.965 & & 0 & & 7 & 7 \\
\hline 1.966 & & 1 & & 10 & 11 \\
\hline 1.967 & & 2 & & 22 & 24 \\
\hline 1.968 & & 0 & & 29 & 29 \\
\hline 1.969 & & 1 & & 38 & 39 \\
\hline 1.970 & & 7 & & 31 & 38 \\
\hline 1.971 & & 23 & & 25 & 48 \\
\hline TOTAL & & 44 & & 189 & 233 \\
\hline PORCENTA JE & & 8.88 & & 81.12 & 100.00 \\
\hline
\end{tabular}


CUADRO N24

MOTIVO DE CONSULTA DE 233 PACIENTES CON CAR. CINOMA CERVICAL IN-SITU-HOSPITAL UNIVERSITARIO SAN VICENTE DE PAUL. MEDELLIN 1.958 - 1.971

\begin{tabular}{|l|c|c|}
\hline MOTIVO DE CONSULTA & No PACIENTES & POR CENTA JE \\
\hline FLUJO & 100 & 42.92 \\
HEMORRAGIA VAGINAL & 52 & 22.32 \\
PROLAPEO & 17 & 7.30 \\
OTRA PATOLOGIA & 2 & 8.86 \\
ASINTOMATICA & 62 & 26.60 \\
\hline TOTAL & 233 & 100.00 \\
\hline
\end{tabular}

CUADRO NE 5

ASPECTO CLINICO DEL CUELLO UTERINO DE 233 PACIENTES CON CARCINOMA IN-SITU-HOSPITAL UNIVERSITARIO SAN VICENTE DE PAUL. MEDEWN 1.958-7I

\begin{tabular}{|c|c|c|}
\hline ASPECTO & NO PACIENTES & PORCENTAJE \\
\hline CUELLO UTERINO & 70 & 30.04 \\
SENO & 145 & 62.23 \\
EROSIONADO & 12 & 5.15 \\
ULCERADO & 3 & 1.29 \\
MASA PROLIFERATIVA & 3 & 1.29 \\
POLIPO CERVICAL & 233 & 100.00 \\
\hline TO TAL & & \\
\hline
\end{tabular}


te estaban completamente sanos (Cuadro N. 5); es importante por lo tanto tomar citología a cualquier cérvix por sano que parezca a la vista del examinador.

\section{Citología cérvico-vaginal previa al} diagnóstico de carcinoma in situ. Como era de esperarse en las pacientes que presentaron carcinoma in situ la citología fue positiva en un $88,8 \%$ entre los grados IV y V, y el 5,15\% presentaron citologías grado III persistente, lo que refleja la gran fidelidad de este examen para detectar el cáncer pre-invasivo aún en los cuellos sanos (Cuadro N: 6) (27, 30, 32 ).

Relación entre la citología y el aspecto macroscópico del cuello. El Cuadro № 7 trata de buscar si existe alguna relación entre las lesiones clínicas del cuello uterino y las citologías tomadas antes de establecer el diagnóstico definitivo de Carcinoma Intraepitelial y encontramos los siguientes: En los cuellos sanos el $92,8 \%$ las citologías fueron positivas (grados IV - V), en los cuellos erosionados el $88,9 \%$ de las citologías fueron positivas, cuando el cuello estaba ulcerado el porcentaje de positividad fue de $83,3 \%$ y cuando el cuello presentaba masa proliferativa la citología fue positiva en el $66,66 \%$ pero de esta última la tercera parte no se le tomó citología. Esto nos indica que existe una pequeña diferencia inversa entre la lesión del cuello y el índice de positividad de la citología, pero también nos dice que por muy lesionado que esté el cuello la citología presenta un alto porcentaje de efectividad.

Vemos entonces que cualquier tipo de cuello, ya sea sano, erosionado o ulcerado, de los que encontramos, comunmente, puede dar citologías posi- tivas y por lo tanto tener un posible carcinoma in situ.

\section{Resultado anatomopatológico de} la pieza quirúrgica según la base del diagnóstico de biopsia múltiple o biopsia y cono. El Cuadro № 8 nos revela que de los casos de carcinoma in itu sdiagnosticados por biopsia múltiple se encontraron el 2,2\% de carcinoma invasor en la pieza definitiva; las pacientes en las que el diagnóstico definitivo de carcinoma in situ se efectuó por conización no se encontró ningún caso de invasión.

En un trabajo sobre carcinoma in situ hecho en Medellín con pacientes del Hospital Universitario San Vicente de Paúl y del Instituto Colombiano de Seguros Sociales (28) los autores encontraron carcinoma invasor en la pieza definitiva, sin hacer conización, (sólo con biopsia múltiple), en el 0,5\% de los casos. Otro trabajo sobre carcinoma hecho en el Metropolitan Hospital Medical Center de New York (29) informa de algunas ventajas de la biopsia múltiple obviando el cono para disminuir la morbilidad y los costos del mismo.

Nos hacemos entonces la pregunta, y queremos dejarle la inquietud al lector de si los porcentajes de invasión de $0,5 \%$ a $2,2 \%$ que se obtienen con la biopsia múltiple, justificarían agregar mayor morbilidad, costos y traumatismos sicológicos a la paciente al efectuar el cono.

El carcinoma residual se encontró en el $34 \%$ de las pacientes a las que se les efectuó cono, cifra que está cercana a la encontrada por otros autores $(7,27,28,29,30,33)$. Dadas las cifras anteriores tan alats de carcinoma residual hacen del cono un tratamiento inadecuado para esta lesión. 
DISTRIBUCION DE LAS CITOLOGIAS CERVICALES PREVIAS AL DIAGNOSTICO DE CARCINOMA IN-SITU. HOSPITAL UNIVERSITARIO SAN VICENTE DE PAUL. MEDELLIN $1.958-1.971$

\begin{tabular}{|c|c|c|}
\hline CITOLOGIA & NO PACIENTES & PORCENTAJE \\
\hline GRADOIII PERSISTENTES & 12 & 5.15 \\
GRADO IV & 144 & 61.80 \\
GRADO V & 63 & 27.03 \\
OTROS GRADOS & 1 & 0.43 \\
SIN DATOS & 3 & 1.29 \\
SIN CITOLOGIA & 10 & 4.30 \\
\hline TO TAL. & 233 & 100.00 \\
\hline
\end{tabular}

Se encontraron 7 pacientes embarazadas a las cuales se les efectuó conización. Cuatro de ellas con carcinoma residual, lo que nos dá un porcentaje muy alto si lo comparamos con las nó embarazadas, dato que está de acuerdo con algunos autores (34). Pero no hay acuerdo entre los diversos trabajos consultados a este respecto de carcinoma in situ y embarazo $(11,35,36,37)$.

Merece la pena mencionar el hecho de que la citología en la embarazada es tan fiel como en la no gestante, discusión que ya no existe porque un buen citólogo sabe diferenciar cuáles son los cambios de la gestación y cuáles los de las células neoplásicas $(38,39,40)$.

En lo que se refiere a las pacientes en embarazo con carcinoma in si- tu el seguimiento y tratamiento tiene algunas diferencias según diversos autores; todos están de acuerdo en que el parto debe ser por vía vaginal $(10,11,36)$, pero difieren en cuanto el momento del tratamiento definitivo; pasado el parto se les toma la citología y si ésta es negativa no la intervienen; en cambio, si la citología es positiva le efectúan histerectomía con resección del manguito vaginal (10). Otros intervinieron a todas sus pacientes entre 6 y 18 meses después del parto (11).

Multiparidad y carcinoma in situ. Se ve una relación entre multiparidad y carcinoma in situ en el Cuadro № 9, lo que está de acuerdo con la mayoría de los autores $(1,3,27$, 41). El $93 \%$ de nuestras pacientes 
RELACION ENTRE EL ASPECTO CLINICO DEL CUELLO UTERINO Y LA CITOLOGIA CERVICAL EN 233 PACIENTES CON CARCINOMA INTRAEPITELIAL. HOSPITAL UNIVERSITARIO SAN VICENTE DE PAUL. MEDELLIN 1.958-7I

\begin{tabular}{|l|c|c|c|c|c|}
\hline \multirow{2}{*}{$\begin{array}{l}\text { RESULTADO } \\
\text { CITOLOGIA }\end{array}$} & \multicolumn{5}{|c|}{ ASPECTO CUELLO UTERINO } \\
\cline { 2 - 6 } & SA NO & EROSIONADO & ULCERADO & $\begin{array}{c}\text { MASA PRO- } \\
\text { LI FERATIVA }\end{array}$ & POLI POS \\
\hline III PERSISTENTE & 2.86 & 6.21 & 8.33 & - & - \\
IV PERSISTENTE & 65.71 & 61.38 & 58.33 & 33.33 & 33.33 \\
V PERSISTENTE & 27.14 & 27.59 & 25.00 & 33.33 & - \\
OTRO GRADO & - & - & - & - & 33.33 \\
SIN DATOS & - & 0.69 & - & - & - \\
SIN CITOLOGIA & 4.29 & 4.14 & 8.33 & 33.33 & 33.33 \\
\hline TOTA L & 100.00 & 100.01 & 99.99 & 99.99 & 99.99 \\
\hline NUMERO & 70 & 145 & 12 & 3 & 3 \\
\hline
\end{tabular}

tenían entre 3 y más hijos, con un promedio de hijos de 7.6.

Meses-mujer de observación. En el Cuadro № 10 que trata sobre el seguimiento de las pacientes vemos que hubo 7.294 .5 meses-mujer-observación lo cual es considerado como una cifra récord en nuestro medio. Estas pacientes se controlaron en su mayoría de la siguiente manera: una primera cita al mes de intervención, luego cada 3 meses en el primer año y de allí en adelante cada 6 meses. De las pacientes localizadas por carta y otro medio de comunicación asistieron a control, las que no vinieron quedaron fuera de este cuadro, pero sí dentro del Cuadro № 13.
Tratamiento. No fue uniforme el tratamiento en todas las pacientes estudiadas debido al gran período de tiempo que abarca y a la evolución del mismo de acuerdo a la época; en general a la mayoría se les hizo histerectomía total y resección del tercio superior de vagina como lo aconsejan muchos autores (12, 13, 14, $15,16,17,18,19)$. Cuando se hizo como la histerectomía se llevó a cabo entre las primeras 48 horas o después de 6 semanas para evitar las complicaciones mencionadas en otros trabajos $(33,42)$. En el Cuadro $N$ ㅇ 11 nos llama la atención el 4,3\% de cirugía radical que se efectuó en los primeros años de este trabajo, cuando no estaba bien claro el tratamien- 
RESULTADO ANATOMOPATOLOGICO DE LA PIEZA QUIRURGICA (UTERO) SEGUN LA BASE DEL DIAGNOSTICO DE BIOPSIA MULTIPLE O BIOPSIA Y CONO DE LAS PACIENTES CON CARCINOMA INTRAEPITELIAL. DISTRIBUCION PORCENTUAL. HOSPITAL UNIVERSITARIO SAN VICENTE DE PAUL.

MEDELLIN $1.958-1.971$

\begin{tabular}{|l|c|c|}
\hline \multirow{2}{*}{$\begin{array}{c}\text { RESULTADO DE ANA- } \\
\text { TOMIA PATOLOGICA. }\end{array}$} & BASE DEL & DIAGNOSTICO \\
\cline { 2 - 3 } & BIOPSIA MULTIPLE & BIOPSIA CONO \\
\hline NEGATIVA & 4.44 & 65.43 \\
DISPLASIA MODERADA & 2.22 & - \\
DISPLASIA SEVERA & 2.22 & - \\
CIE & 82.22 & 34.04 \\
MICRO INVASOR & 6.67 & 0.53 \\
INVASOR & 2.22 & - \\
\hline TO TAL & 99.99 & 100.00 \\
\hline NUMERO & 45 & 188 \\
\hline
\end{tabular}

CUADRO N:9

DISTRIBUCION DE PACIENTES CON CARCINOMA INTRAEPITELIAL SEGUN EL NUMERO DE PARTOS EN 233 PACIENTES DEL HOSPITAL UNIVERSITARIO SAN VICENTE DE PAUL. MEDELLIN $1.958-1.971$

\begin{tabular}{|l|c|c|}
\hline PARIDAD & No CASOS & PORCENTAJE \\
\hline PRIMIPERAS & 7 & 3.00 \\
SECUNDIPERAS & 8 & 3.43 \\
MULTIPARAS ( 3 a 5 ) & 62 & 26.61 \\
MULTIPARAS (5y más) & 155 & 66.53 \\
DES CONOCIDAS & 1 & 0.43 \\
\hline TOT AL & 233 & 100.00 \\
\hline
\end{tabular}


TIEMPO DE SEGUIMIENTO Y MESES-MUJER DE OBSERVACION DE 233 PACIENTES CON CARCINOMA IN-SITU, DESDE EL MOMENTO DE SU INTERVENCION QUIRURGICA. HOSPITAL UNIVERSITARIO SAN VICENTE DE PAUL. MEDELLIN 1.958-1.972

\begin{tabular}{|l|c|c|c|}
\hline $\begin{array}{c}\text { SEGUIMIENTO } \\
\text { MESES }\end{array}$ & No PACIENTES & PORCENTAJE & $\begin{array}{c}\text { MESES MUJER DE } \\
\text { OBSERVACION }\end{array}$ \\
\hline MENOS DE I & 6 & 2.57 & 0.5 \\
I A MENOS DE 3 & 18 & 7.73 & 27.0 \\
3 A MENOS DE 6 & 20 & 8.60 & 74.0 \\
6 A MENOS DE 9 & 20 & 8.60 & 138. \\
9 A MENOS DE 12 & 17 & 7.30 & 166.0 \\
I2A MENOS DE 24 & 39 & 16.73 & 659.0 \\
24 A MENOS DE 36 & 31 & 13.30 & 905.0 \\
36 A MENOS DE 48 & 31 & 13.30 & 1.265 .0 \\
48 A MENOS DE 60 & 15 & 6.43 & 787.0 \\
60 Y máS & 36 & 15.44 & 3.273 .0 \\
\hline T O T A L & 233 & 100.00 & 7.294 .5 \\
\hline
\end{tabular}

to. Hay un $3 \%$ de pacientes con conización únicamente debido a que deseaban tener más hijos o no querían quedar sin útero para no crearse problemas sociofamiliares.

Complicaciones de la histerectomía. No hubo complicaciones en el $85,4 \%$ de las intervenciones (Cuadro № 12 ). Los pocos problemas operatorios sobre las vías urinarias (ligadura de uréter y fístula urinaria) se presentaron en la histerectomía radical. Las otras complicaciones infecciosas: de la cúpula vaginal, de la pared abdo- minal y de las vías urinarias son las comúnmente encontradas en la cirugía ginecológica. La única muerte que se presentó en el postoperatorio inmediato fue en el año de 1958 en una paciente a quien se le hizo histerectomía radical.

Estado de las pacientes el 31 de Diciembre de 1971. (Cuadro № 13). Vivas: el $77,68 \%$. Este porcentaje de seguimiento se consiguió merced a comunicaciones dirigidas a las pacientes ya sea personalmente o por interme- 


\section{DISTRIBUCION DE PACIENTES CON CARCINOMA IN -} SITU DE ACUERDO CON EL TIPO DE INTERVENCION PRACTICA EN LOS 233 CASOS. HOSPITAL UNIVERSITARIO SAN VICENTE DE PAUL. MEDEUN 1.958-1.97I

\begin{tabular}{|c|c|c|}
\hline TIPO DE INTER VEN CION & $\mathrm{N} \cong$ PACIENTES & PORCENTAJE \\
\hline $\begin{array}{l}\text { HISTERECTOMH TOTAL ABDOMINAL YRESAC - } \\
\text { CION DEL } 1 / 3 \text { SUPERIOR DE VAGINA }\end{array}$ & 173 & 74.25 \\
\hline HISTERACTOMIA RADICAL & 10 & 4. 30 \\
\hline $\begin{array}{l}\text { ANEXOHISTERACTOMIA Y RESACCION DEL } \\
\text { I/ } 3 \text { SUPERIOR DE VAGINA }\end{array}$ & 25 & 10.73 \\
\hline $\begin{array}{l}\text { HISTERACTOMIA VAGINAL,RESACCION DEL } \\
\text { I/3 SUPERTOR DE VAGINA Y PLASTIAS }\end{array}$ & 15 & 6.43 \\
\hline EXTIRPACION DE CUELLO RESTANTE & 2 & 0.86 \\
\hline CONIZACION UNICAMENTE & 7 & 3.00 \\
\hline RADIUM & 1 & 0.43 \\
\hline$T O T A L$ & 233 & 100.00 \\
\hline
\end{tabular}

dio de personas influyentes en la comunidad donde viven.

Muertas: 2 pacientes; una falleció en el postoperatorio inmediato después de una histerectomía radical; la otra murió 2 meses después por una leucemia mieloide crónica.

Pérdidas en esa fecha: el 21,46\%, o sea 50 pacientes, las cuale sfue difícil localizarlas por la vida nómada que llevan debido a sus escasos recursos económicos. El seguimiento del $77,68 \%$ indica un gran esfuerzo pues no tenemos un servicio social organizado que se encargue de estas pacientes.

\section{Recidivas}

De las pacientes con seguimiento tenemos 2 casos para analizar: a una paciente se le había hecho anexohisterectomía el 22 de Octubre de 1964 y en Enero de 1970 se le aplicó cobalto para un carcinoma invasor de cúpula; a la otra paciente se le hizo anexohisterectomía en Noviembre de 1965 y a los 8 meses se le encontró 
CUADRO № 12

COMPLICACIONES INTRA Y POST-OPERATORIOS DE 233 PACIENTES CON CARCINOMA INTRAEPITELIAL — HOSPITAL UNIVERSITARIO SAN VICENTE DE PAUL - Medellín 1958-1971

\begin{tabular}{|l|c|}
\hline \multicolumn{1}{|c|}{ COMPLI CA CIONES } & PORCENTAJE \\
\hline MUERTE EN PRIMERAS 24 HORAS & 0.43 \\
INFECCION DE CUPULA VAGINAL & 3.43 \\
INFECCION DE PARED ABDOMINAL & 1.72 \\
INFECCION DE VIAS URINARIAS & 2.57 \\
FISTULA URINARIA & 0.43 \\
HEMORRAGIA DE CUPULA & 1.29 \\
LIGADURA DE URETER & 0.43 \\
RUPTURA ACCIDENTAL DE VEJIGA EN ELACTO OPERATORIO & 0.43 \\
OBSTRUCCION INTESTINAL POR BRIDAS & 0.43 \\
SIN COMPLICACIONES & 85.41 \\
NO A PLICABLE ( NO hISTERECIOMIO). & 3.43 \\
\hline T O T A L & 100.00 \\
\hline
\end{tabular}

CUADRO N: 13

ESTADO DE LAS PACIENTES EL 31 DE DICIEMBRE DE 1971 DE LOS CASOS CON CARCINOMA IN SITU DEL CUELLO UTERINO - HOSPITAL UNIVERSITARIO SAN VICENTE DE PAUL Medellín - 1958-1971

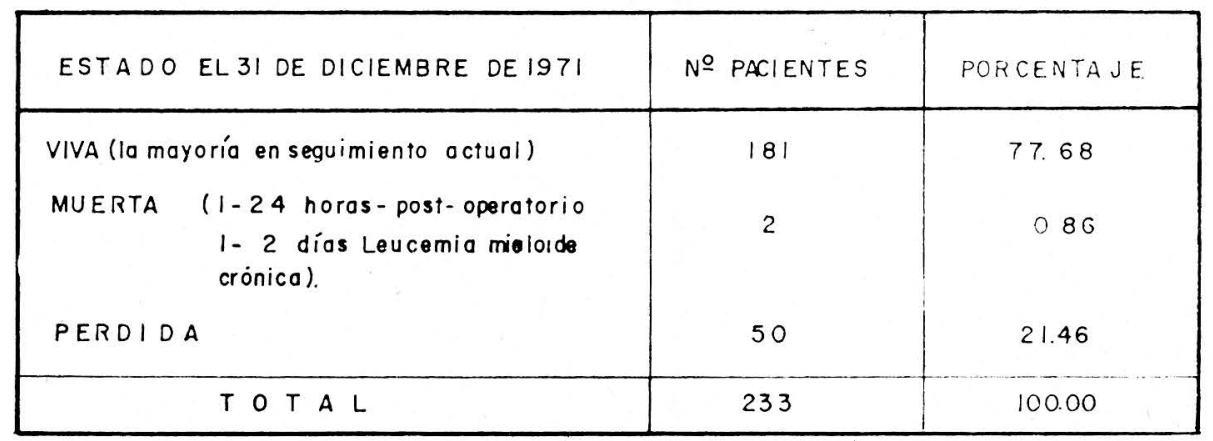


carcinoma intraepitelial de la cúpula y se trató con Radioterapia. No tenemos muy claro si estos dos casos son una continuación de la evolución del carcinoma in situ como ya fue anotado antes $(3,7,8)$ en los que se quedó algún foco, de los multicéntricos que dan origen al carcinoma intraepitelial, o eran zonas nuevas de carcinoma.

\section{Conclusiones}

Estudiamos el carcinoma in situ en nuestro medio dándole a la citología vaginal la importancia que tiene como medio de detección del mismo.

Se hace notar también la gran diferencia que existe entre el número tan alto de carcinoma invasor en el Hospital Universitario San Vicente de Paúl y el carcinoma in situ del cuello uterino y el papel que deben desempeñar los médicos en general para reducir esta diferencia. Es intersante y nos llena de optimismo el hecho de que la curva de casos diagnosticados de carcinoma in situ va creciendo cada vez más.

Capítulo aparte merece el estudio del carcinoma in situ en la embarazada; en esta paciente también se debe imponer la citología de rutina en la consulta prenatal de manera similar a como se hacen los otros estudios de laboratorio; dejar pasar nueve meses o más para tomar una citología es darle oportunidad de avanzar a una lesión premaligna. Se conocen factores que están presentes en la mayoría de las pacientes con carcinoma pre-invasor como son la mayor paridad y las décadas 4a y 5a de la vida, casos en los cuales hay que hacer énfasis en la pesquisa de este tumor.

El tratamiento debe hacerse pensando en que esta lesión pre-invaso- ra puede convertirse en invasora; se acepta generalmente que la terapia definitiva y mejor es la histerectomía total con resección de un manguito vaginal de por lo menos $2 \mathrm{cms}$., teniendo en cuenta que la lesión residual es alta y que el cono es tratamiento inadecuado; hay que hacer excepciones a la regla anterior: En las pacientes jóvenes, que quieren tener más hijos, etc.

Pensamos también que puede haber algunos casos en que la biopsia múltiple será el paso previo a la histerectomía cuando se reúnan condiciones, sabiendo que el cono puede aumentar la morbilidad y los costos del tratamiento $(4,3)$.

El tratamiento más aceptado por la generalidad de los autores, es el ya mencionado atrás, se ha unificado para el carcinoma in situ; en los últimos años ya no se han hecho más histerectomías ampliadas y el cono sólo se considera como un paso diagnóstico.

Una de las principales dificultades para el control de nuestros casos es el tipo de paciente que asiste al hospital: Son personas de escasos recursos económicos que cambian frecuentemente de habitación, de barrio y de ciudad; necesitamos un servicio socia Icon disponibilidad de medios de transporte y personas para localizar esta gente nómada.

Nosotros nos valimos de cartas y algunas veces de emisoras para mejorar el seguimiento y alcanzar el que tenemos.

Nunca haremos el suficiente énfasis en llamar la atención sobre el diagnóstico precoz de estas lesiones pre-malignas que tienen una curación del $100 \%$ cuando son bien tratados. Suponemos que ésta debe ser una de las metas de la ginecología actual. 


\section{Recomendaciones:}

1. Hacer examen genital a las pacientes que acuden a nuestra consulta.

2. Tomar citología en cualquier tipo de paciente femenino sin tener en cuenta edad, paridad, embarazo, etc. mereciéndole más la multípara y el estar comprendida entre los 30 y 50 años de edad.

3. Si la citología es positiva, tomar biopsia múltiple previa prueba de Schiller para localizar mejor la zona patológica del cérvix. Continuar luego con el cono o la histerectomía según las consideraciones de cada caso.

4. Si se trata de una paciente embarazada con citología positiva, hacer biopsia y continuar con la conización, si fuere necesario, para aclarar el diagnóstico, sin temerle a esto sprocedimientos.

5. El seguimiento y control deben hacerse en lo posible en el $100 \%$ de los casos por un máximo tiempo para tratar precozmente cualquier recidiva.

6. Informar a la paciente que esta lesión se cura en un $100 \%$ cuando es tratada adecuadamente y que la palabra Cáncer in situ que se le asigna no tiene el significado que se le atribuye generalmente.

7. Tener en cuenta que muchas de nuestras pacientes son grandes multíparas donde hay una relación directa con esta entidad.

8. Debe existir una estrecha colaboración entre Citólogo, Patólogo y Clínico. Si el patólogo es al mismo tiempo citólogo, las ventajas se suman en la precisión diagnóstica.

\section{Resumen}

Se hace un estudio sobre 233 pacientes con carcinoma in situ en el Hospital Universitario San Vicente de Paúl de Medellín, Colombia, durante los últimos 13 años. Se hace énfasis sobre la toma de la citología en toda paciente ginecológica incluyendo la consulta prenatal. Se analizan los datos encontrados en cada paciente, y se dan algunas recomendaciones.

\section{Summary}

A study was performed on 233 patients with carcinoma in situ at Hospital Universitario San Vicente de Paúl in Medellín, Colombia, during the past 13 years. Emphasis is made on the performance of cystology to all gynecological patients, including prenatal examinations. An analysis is made on the data encountered in each patient and several recommendations are made.

\section{BIBLIOGRAFIA}

1 CARTER B. et al. Clinical problems in stage $O$ (intraepithelial) cancer of the cervix. Amer. J. Obst. Gynec. 71: 634, 1956.

2 MACFARLANE C. Vaginal cytology which patients should have priority? Amer. J. Obstet. Gynec. 77: 1151, 1959.

3 REAGAN J. W. and W. BUDD MENTZ. Génesis del carcinoma del cuello uterino. Clin. Obst. y Ginec. Pág. 883, Dic. 1967.

4 RICHART R. M. and BARRON B. A. Followup study of patients with cervical dysplasia. Am. J. Obstet. \& Gynec. 105: 386, 1969.

5 VILLALBA R. Diagnóstico del $\mathrm{Ca}$. in situ del cuello uterino. Rev. Obst. Gin. Venezuela. 24 : 535,1964

6 JORDAN G. El problema del carcinoma intraepitelial del cuello uterino. Universitas Médica. 1: 39, 1953.

7 CAMPOS J. Diagnóstico y naturaleza del carcinoma in situ. Rev. Mex. Cir. Ginec. Cáncer. 33: 200, 1965. 
8 RICHART R. M. Cervical neoplasia in pregnancy. Amer. J. Obstet. Gynec. 87: 474, 1963.

9 MUSSEY E. and DECKER D. G. Intraepithelial carcinoma of the cervix in association with pregnancy. Amer. J. Obst. Gynec. 97: $30,1967$.

10 STROMME W. B. Preclinical carcinoma and dysplasia of the cervix associated with pregnancy. Am. J. Obst. \& Gynec. 105: 1006, 1008, 1969.

11 HANLESS J. F. Carcinoma of the cervix in pregnancy. Am. J. Obst. Gynec. 110: 173, 1971.

12 FUNNELL J. D. and J. A. MERRILL. Recurrence after treatment of carcinoma in situ of the cervix. Surg. Gynec. Obst. 117: 15, 1963.

13 CACERES E. Importancia de la colpocitología en programas de planificación familiar. Rev. Col. Cbst. y Gin. 23: 55, 1972.

14 COPPLESOMM, REID B. Mnaagement of cervical carcinoma in situ. Lancet, 2: 873 , 1968.

$15 \mathrm{CHAD}, \mathrm{S}$. Conization in evaluation an treatment of cervical neoplasia. Am. J. Obst. and Gynec. 103: 574, 1969.

16 KOTTMATER H. L. Current treatment of carcinoma of cervix. Am. J. Obst. Gynec. 76: 243, 1958.

17 KRIEGER J. S. and MarCORMACK L. J. Graded treatment for in situ rarcinoma of uterine rervix. Am. J. Obst. Gynel. 107: 171, 1968.

18 NELSON J. H. and J. E. HALL. Detection, diagnostic and treatment dysplassia and early carcinoma of cervix. Cáncer. 20: 150, 1970.

19 NOVAK JONES. Tratado de ginecología. Carcinoma del cuello. 12. p. 243, 1971.

20 BAQUERO E., TUBERQUIA J. C. Prolapso genital en la mujer joven tratado con suspensión con dacrón. Próximo a publicarse. Rev. Col. Obst. Ginec. Dic. 1972.

21 GRANER, H. La colposcopia en la práctica. Barcelona, Editorial Labor. 1963.

22 BOLTEN K. Colposcopia práctica en el cáncer cervical y vaginal incipientes. Clínicas Obstétricas y Ginecológicas. Pág. 808, Dic. 1967
23 NARRATIL, E. et al. Simultaneous colpocopy and citology used in screennig for carcinoma of the cervix. Am. J. Obst. Gynec. 75: 1292, 1958.

24 NUÑEZ MONTIEL, et al. Detection of Early endocervical carcinoma using colposcopy. Obst. \& Gynec. 35: 781, 1970.

25 ORG. MUND. SALUD. Ser. Inf. Tecn. 1971, NNo 473 Pág. 32.

26 RICHART R. M. Historia natcral de la neoplasia cervical intraepitelial. Clin. Obst. y Gynec. Pág. 747, Dic. 1967.

27 JORDAN G. y col. Carcinoma in situ del cérvix. Rev. Col. Obst. y Ginec. 22: 71, 1971.

28 URIBE J. Y JUBIZ A. Tratamiento de las lesiones premalignas del cuello uterino. Estudio de 271 casos. Trabajo presentado en el IX Congreso Colombiano de Obstetricia y Ginecología. Bucaramanga, Colombia, 1972.

29 SABATELLE R. et al. Cervical biopsy versus conization. Cáncer. 23: 633, 1969.

30 BURBANO F. y BOTERO G. Carcinoma intraepitelial. Tribuna Médica. 26: 58, Febrero 2, 1970.

31 FERENCZY $A$. et al. Endometrial involvement by cervical carcinoma in situ. Amer. J. Obstet. Gynec. 110: 591, 1971.

32 FLUHMANN C. F. Carcinoma in situ and the transitional zone of the cervix uteri. Obst. \& Gynec. 16: 424, 1960.

33 PEDERSON B. L. and JEFFRIES F. W. Cervical carcinoma in situ. Obst. \& Gynec. 26 : 725, 1965.

34 MAY, S. Exfoliative Citology, early carcinoma of the cervix, pregnancy and fertility. Acta Cytológica. 6: 547, 1962.

35 STONE et al. Cervical carcinoma and pregnancy. Am. J. Obst. Gynec. 93: 479, 1965.

36 GUZMAN R. D. Y FONNEGRA A. Carcinoma in situ y embarazo. Rev. Col. Obst. y Gin. 23: $35,1972$.

37 RUTLEDGE, G. E. Jr. et al. Cervical Displasia and carcinoma in pregnancy. Obst. \& Gynec. 19: 351, 1962.

38 SLATE T. A. et al. Am. J. Obst. \& Gynec. 74: 344, 1956.

39 FERGUSON and CAVANGH. Am. J. Obst. \& Gynec. 74: 344, 1956. 
40 AYRE, J. E. Obst. \& Gynec. 76: 780 1958.

41 VILLARREAL-CACERES. Carcinoma de cueIlo uterino. Rev. Col. Obst. y Gin. 12: 570, 1961.

42 MALIMAK, L. R., JEEFREY, R. A. and DUNN, W. J. The conization hysterectomy time interval. A. Clinical and pathological study. Obst. \& Gynec. 23: 317, 1964.

43 GRIFFYTHS C. T., AUSTIN J. H., and COUNGE, P. A. Punch Biopsy of the cer- vix. Amer. y Obstet. Gynec. 88: 695 1964.

44 ISAZA M., GUSTAVO. Diagnóstico del Cáncer preclínico del útero. Antioquia Médica, Medellín, 1: 331, 1950.

45 ISAZA M. GUSTAVO. "Carcinoma incipiente del cérvix uterino". Antioquia Médica. Medellín. 4: 7, 1954.

46 JORDAN A., GERMAN. "Carcinoma intraepitelial del Cuello Uterino". Rev. Col. Obst. y Ginec. Bogotá, 5: 159, 1954 